\title{
SMIMO Radar: MIMO Radar with Subarray Elements of Phased-Array Antenna
}

\author{
Syahfrizal Tahcfulloh ${ }^{1}$
}

\begin{abstract}
Unlike Phased-MIMO Radar (PMIMO) which employs overlapping equal subarrays (OES) only on the transmit (Tx), Subarray-MIMO (SMIMO) radar utilizes the combination of subarrays, both in the transmit $(\mathrm{Tx})$ and receive $(\mathrm{Rx})$. SMIMO radar is MIMO radar with subarray elements serving as PhasedArray (PA). It simultaneously combines the primary advantages of PA and the MIMO radar; they are high directional gain and high diversity gain. High directional gain is beneficial to improve the range target, while high diversity gain is beneficial to improve the number of target detection. The use of the subarray methods in the Tx-Rx array could be configured such as in overlapping subarray (OS), non-overlapping subarray (NOS), equal subarray (ES), unequal subarray (US), and/or the combination of all configurations. Various configurations in $\mathbf{T r}$ Rx would determine the performance of radar, such as the number of virtual arrays, the maximum number of target detections, the detection accuracies, and the angular resolutions along with its effectivity compared to PA, MIMO, and PhasedMIMO radar. Numerical results and simulation showed that SMIMO provided higher flexibility than other radars by configuring $T x-R x$ to easily adapt to various changes of target conditions and their surroundings.
\end{abstract}

Keyword-Angular Resolution, MIMO Radar, Number of Detectable Targets, Phased-array, Subarray, Target Detection, Virtual Array.

\section{INTRODUCTION}

In line with the development and trend of multi-antenna radar technology to detect multi-targets and maximum range, a radar with high detection capability and flexibility supported by detection accuracy and high angular resolution is needed. In its implementation, especially as automotive radar, a radar with high accuracy and resolution of multi-target detection is needed [1]. Prevalent forms of multi-antenna radar are Phased-Array (PA) and MIMO radars. The PA radars are antennas that are being fed by adjusting their phase difference to obtain high directional gain in certain directions [2], [3], so that the small angular resolutions limit the number of target detections. The number of target detections is increased by implementing MIMO radar as it utilizes many transmission signals that are orthogonal to each other to obtain many echo signals reflected by multi-targets [4]-[7], yet they have low directional gains.

Phased-MIMO (PMIMO) radars resolve the problems faced by PA and MIMO [8]-[11] by forming subarrays that equally overlap on the transmit array (Tx). As the results, they can

\footnotetext{
${ }^{1}$ Department of Electrical Engineering of Universitas Borneo Tarakan, Jln. Amal Lama No.1 Tarakan 77123 INDONESIA (phone: +628115307023; fax: 0551-2052558; e-mail: rizalubt@gmail.com)
}

simultaneously utilize the main advantages of PA radars, namely high directional gain, and the main advantages of MIMO radar, namely high diversity gain, to increase the number of target detections and ranges. Hereafter, this radar is called PMIMO-Tx.

References [12]-[14] on Full-PMIMO radars (FPMIMO) have stated that the use of overlapping subarrays in both the Tx and receive $(\mathrm{Rx})$ arrays increase the maximum number of detections and angular resolutions. However, the number of elements in each Tx-Rx subarrays is equal. Henceforth, this radar is called PMIMO-Tx-Rx.

This paper will present the primary contribution of Subarray-MIMO (SMIMO) radars that have not been discussed in previous studies [13], [14] to support the generalizability and flexibility of high multi-target detection. The subarrays can be conditioned as whether a) the number of elements is varied and/or is equal and b) overlapping and/or non-overlapping on $\mathrm{Tx}-\mathrm{Rx}$ so that they can form a PA, MIMO, PMIMO-Tx, PMIMO-Tx-Rx, and other radar variations. The conditions of the subarrays in the SMIMO radar increase the degree of freedom (DoF) compared to other radars because other radars are special conditions of this radar.

SMIMO radar performance is presented in the virtual array (VA) size, the maximum number of targets, angular resolutions, and the maximum complex of echo targets. The results showed that SMIMO radar had high flexibility in varying the configuration of the number of elements in the subarrays, the number of subarrays, the overlap/non-overlap adjusting the condition of the target detection at that time. Primarily if they were implemented using a programmable platform for radar system design such as USRP [15].

The subsequent section, Section II, will present the model of the SMIMO radar system and signal. Meanwhile, Section III will show the mathematical expressions for calculating the $\mathrm{VA}$, the maximum number of detected targets, and the magnitude of the complex amplitude. Section IV will present the results and discussion in the form of numerical calculations and simulations of the performance parameters for the SMIMO radar, as well as the analysis and validation. Section V will contain the conclusions of this paper.

\section{SUBARRAY MIMO RADAR}

\section{A. System Model}

A multi-antenna radar system with a uniform linear array (ULA) consists of $M$ and $N$ total elements in the Tx and Rx. The space between elements in the Tx and Rx is $d_{M}$ and $d_{N}$, respectively. The transmitted signals are the narrowband frequencies with non-dispersive propagation. In the arrays, Tx 

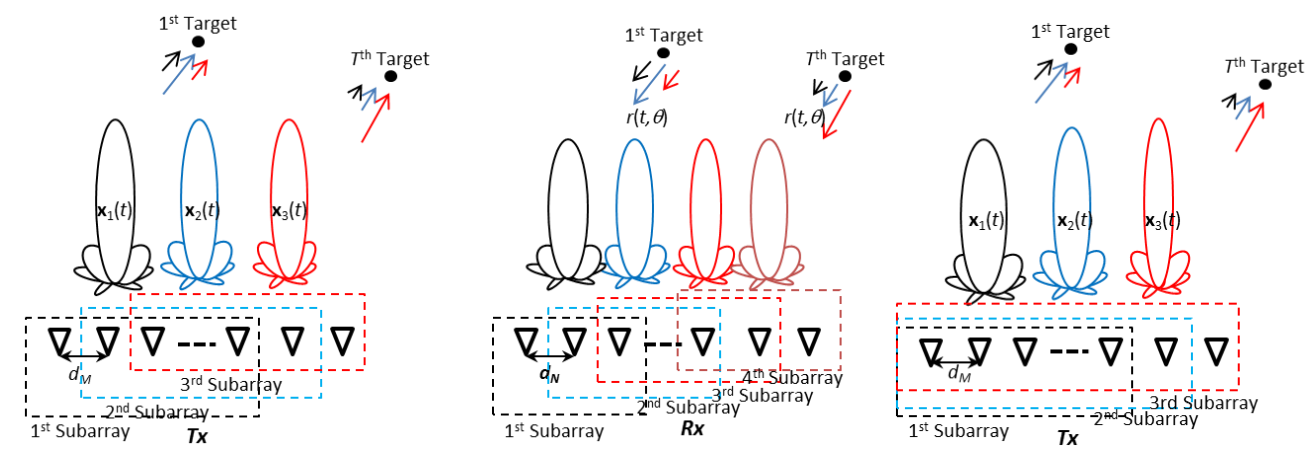

(a)
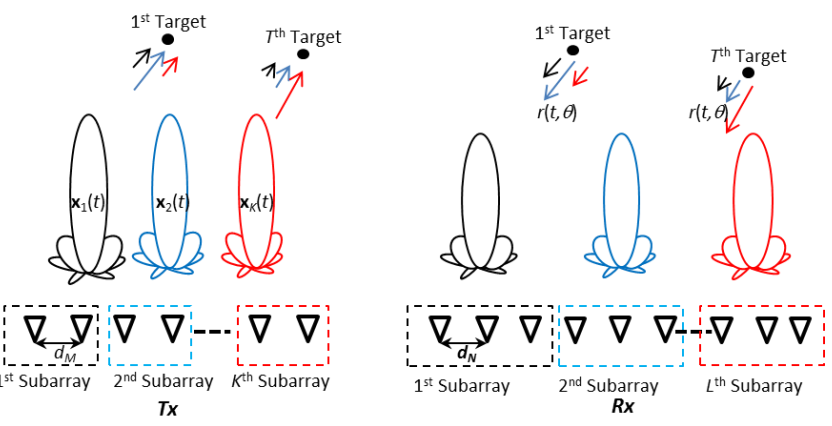

(c)

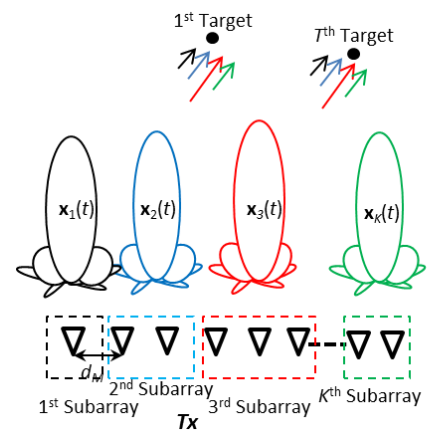

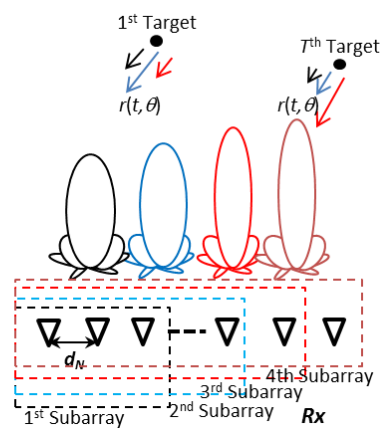

(b)

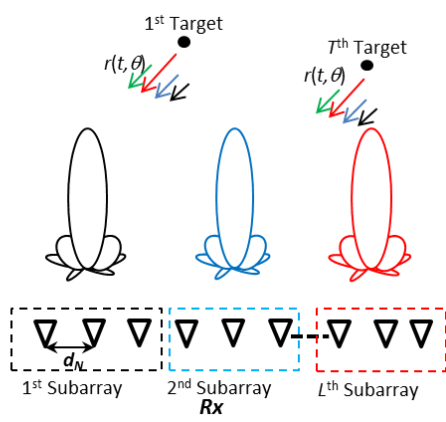

(d)

Fig. 1 Illustration of several Tx-Rx array configurations in the SMIMO radar with subarrays conditions: (a) overlapping with the same element, (b) overlapping with varying element numbers, (c) non-overlapping with the same element, and (d) non-overlapping by varying element number.

and Rx are partitioned into several subarrays, namely $K$ and $L$. Subarrays can follow combinations such as overlapping/nonoverlapping and the amount can be equal/varied, as shown in Fig. 1. The size of subarrays in the $\mathrm{Tx}$ and $\mathrm{Rx}$ is $1 \leq K \leq M$ dan $1 \leq L \leq N$.

Each subarray in both Tx and Rx arrays acts as an element of the MIMO radar array that functions as a directional gain regulator such as PA. Hence, SMIMO radar subarrays act as orthogonal Tx-Rx elements to each other. The configuration of these subarrays promises a combination of directional gain and diversity gain that neither of them has, namely MIMO and PA radars.

Fig. 1 presents several configurations of subarray variations in Tx-Rx arrays in SMIMO radars, such as overlapping/nonoverlappoing and the number of elements of each subarray that is equal or different/varied. Fig. 1(a) is a SMIMO radar configuration with overlapping subarrays. The number of elements for each subarray is the same, it is called an overlapping equal subarray (OES), with $K=3$ in the Tx and $L$ $=4$ in the Rx. This configuration produces three orthogonal signals that are transmitted by three subarrays in the Tx and received by four subarrays operating independently in the $\mathrm{Rx}$ to detect multitargets. Fig. 1(b) shows the conditions between overlapping subarrays in the SMIMO radar which number of elements on each subarray is varied, it is called overlapping unequal subarray (OUS), with $K=3$ in the Tx and $L=4$ in the Rx. As a result, it forms three orthogonal signals which are transmitted by three subarrays in the Tx and received by four subarrays in the Rx. The difference between the OES and
OUS configurations is that the subarrays in the OES configuration have identical radiation forms (beamforming) in the Tx and Rx of each subarray, while the radiation forms in the OUS are not. The difference emerges since the OUS configuration has a various number of antenna elements, where a subarray with many elements will produce higher directional gain.

The configuration of the SMIMO radar subarrays in Fig. 1 (c) is non-overlapping, while the number of each subarray element is equal or non-overlapping equal subarray (NOES) with $K$ subarrays in Tx and $L$ subarrays in the Rx. This configuration produces $K$ orthogonal signals that are transmitted by the Tx array and received by $L$ independent subarrays in the $\mathrm{Rx}$ array for multitarget detection. Meanwhile, Fig. 1(d) shows non-overlapping conditions between subarrays; the number of elements varies in each subarray or non-overlapping unequal subarray (NOUS) with several $K$ subarrays in the Tx and several $L$ subarrays in the Rx. The difference between the NOES and NOUS configurations is that the subarrays in the NOES configuration have identical beamforming in the Tx and Rx of each subarray while subarrays in NOUS does not. The difference is caused by the NOUS configuration which has a various number of antenna elements, as described in Fig. 1(c). In addition to the configuration of subarrays in Fig. 1, SMIMO radar can form other configurations.

The difference between overlapping subarray (OS) and non-overlapping subarray (NOS) will be discussed in Chapter 
IV-A. Meanwhile, this chapter will discuss the VA size and the signal transmitted by semi-orthogonal subarrays, which will directly affect the target's accuracy and detected angular resolution.

\section{B. Signal Model}

The block diagram for SMIMO radar is an extension of the diagram block for FPMIMO/PMIMO-Tx-Rx, which have been presented in [13, Fig. 2]. The differences lie in the number of subarray elements and conditions, of which containing non-overlapping subarray. Subarrays in the Tx radiate orthogonal signals simultaneously toward multitarget. The $k$ th subarray in the Tx array sends $\varphi_{k}(t)$ signals that is independent of one another. Then, the $M$ element in the Tx array will transmit the baseband signal vectors, namely the $k$ th subarrays with $k=1,2, \ldots, M$,

$$
\mathbf{x}_{k}(t)=\sqrt{M / K} \varphi_{k}(t) \mathbf{w}_{k}^{*}
$$

where $(\cdot)^{*}$ is the complex conjugate operator, $M / K$ is the normalization coefficient of power which ensures the transmitted power by the SMIMO radar in one pulse is $M, \mathbf{w}_{k}$ is complex weight vector of normal unit $M$ elements for the $k$ th subarray.

The reflected signal by the target located at $\theta$ in the far field with a reflection coefficient $\beta(\theta)$ is denoted by

$$
\mathbf{r}(t, \theta)=\sqrt{M / K} \beta(\theta) \sum_{k=1}^{K} \mathbf{w}_{k}^{H} \mathbf{a}_{k}(\theta) e^{-j 2 \pi f \tau_{k}(\theta)} \varphi_{k}(t)
$$

where $(\cdot)^{H}$ is the Hermitian transpose operator; $\mathbf{a}_{k}(\theta)$ is the steering vector Tx with $M$ element in the $k$ th subarray; $f$ is the frequency of carrier signal; $\tau_{k}(\theta)$ is the relative delay time of the first element in the $k$ th subarray toward the first element of the first subarray with $\tau_{k}(\theta)=k d_{M} \sin (\theta) / c$, where $c$ is the speed of light in vacuum $\left(\approx 3 \times 10^{8} \mathrm{~m} / \mathrm{s}\right)$.

The coherent vector $\mathrm{Tx}$ and the diversity vector $\mathrm{Tx}$ of the $K$-element can be defined as follows.

$$
\mathbf{c}(\theta)=\left[\begin{array}{llll}
\mathbf{w}_{1}^{H} \mathbf{a}_{1}(\theta) & \mathbf{w}_{2}^{H} \mathbf{a}_{2}(\theta) & \Lambda & \mathbf{w}_{K}^{H} \mathbf{a}_{K}(\theta)
\end{array}\right]^{T}
$$

and

$$
\mathbf{d}(\theta)=\left[\begin{array}{llll}
e^{-j 2 \pi f \tau_{1}(\theta)} & e^{-j 2 \pi f \tau_{2}(\theta)} & \Lambda & e^{-j 2 \pi f \tau_{K}(\theta)}
\end{array}\right]^{T}
$$

Hence, (2) can be simplified to

$$
\mathbf{r}(t, \theta)=\sqrt{M / K} \beta(\theta)(\mathbf{c}(\theta) \operatorname{od}(\theta))^{T} \psi(t)
$$

where $(\cdot)^{T}$ is the transpose operator, $\psi(t)=\left[\begin{array}{lll}\varphi_{1}(t) & \varphi_{2}(t) \ldots\end{array}\right.$ $\left.\varphi_{K}(t)\right]^{T}$ and $o$ is the Hadamard multiplication operator.

Assuming there is $T$ target in the $\left\{\theta_{t}\right\}$ direction with $t=1$, $2, \ldots ., T$, therefore, the complex vector of the signal received by $L$ subarray in the $\mathrm{Rx}$ is

$$
\begin{aligned}
\mathbf{y}(t)=\sqrt{M / K} \sum_{t=1}^{T} \beta_{t}\left(\theta_{t}\right)\left[\mathbf{e}\left(\theta_{t}\right) \operatorname{of}\left(\theta_{t}\right)\right] \\
\cdot\left[\mathbf{c}\left(\theta_{t}\right) \operatorname{od}\left(\theta_{t}\right)\right]^{T} \boldsymbol{\psi}(t)+\mathbf{n}(t)
\end{aligned}
$$

with

$$
\mathbf{e}(\theta)=\left[\begin{array}{llll}
\mathbf{v}_{1}^{H} \mathbf{b}_{1}(\theta) & \mathbf{v}_{2}^{H} \mathbf{b}_{2}(\theta) & \Lambda & \mathbf{v}_{L}^{H} \mathbf{b}_{L}(\theta)
\end{array}\right]^{T}
$$

and

$$
\mathbf{f}(\theta)=\left[\begin{array}{llll}
e^{-j 2 \pi f \tau_{1}(\theta)} & e^{-j 2 \pi f \tau_{2}(\theta)} & \Lambda & e^{-j 2 \pi f \tau_{L}(\theta)}
\end{array}\right]^{T}
$$

where $\mathbf{b}_{l}(\theta)$ is the Rx steering vector with $N$ element in the $k$ th subarray, $\mathbf{v}_{l}$ is complex weight vector of normal unit $N$ elements for the $l$ th subarray, $\mathbf{e}(\theta)$ and $\mathbf{f}(\theta)$ are respectively the coherent vector $\mathrm{Rx}$ and the diversity vector $\mathrm{Rx}$ of the $L$ element, $\mathbf{n}(t)$ is $N$-element vector of white Gaussian noise with zero means.

\section{VIRTUAL ARRAY, MAXIMUM NUMBER OF TARGET, AND COMPLEX AMPLITUDE OF THE SMIMO RADAR ECHO SIGNAL}

The VA formation is the advantage of MIMO radars, which transmit an orthogonal signal, compared to the PA radar [7]. The VA in the PA and MIMO radar highly determine the target detection, angular accuracy, detected angular resolution, and maximum number of detected targets [7], so does the FPMIMO radar with equally overlapped subarrays [14].

The calculation of the VA in the MIMO, PA, FPMIMO or PMIMO-Tx-Rx, and PMIMO-Tx has been proposed in [14, eq. (14)-(17)]. In addition, the maximum number of targets detected by the MIMO and PA radar have been presented in [16, eq. (22) and (25)]. Meanwhile, the PMIMO-Tx-Rx and PMIMO-Tx radar have been presented in [14, eq (32) and (33)]. The calculation of angular resolution calculated from the complex amplitude of the target echo signal in the MIMOTx-Rx, MIMO, PMIMO-Tx, dan PA radars has been discussed in [14, eq. (37), (44)-(46)].

The calculation of the VA, the maximum number of the detected targets, and the complex amplitude of target echo signal in the SMIMO radar are the expansion of the previously conducted study [14]. The number of the VA in the SMIMO and the number of overlapping/non-overlapping or equal/varied subarrays in the Tx and Rx, respectively, can be defined in the $K$ and $L$

$$
E \in[K+L-1, K L] .
$$

The calculation of the maximum number of targets in the SMIMO radar is denoted as

$$
T_{\max } \in\left[\frac{K+L-2}{2}, \frac{K L+1}{2}\right) .
$$

The complex amplitude of the target echo signal in the SMIMO radar is denoted as

$$
\begin{aligned}
& \hat{\beta}(\theta) \\
& =\frac{\sqrt{M / K} \sum_{q=1}^{Q}(\mathbf{e}(\theta) \operatorname{of}(\theta))^{H} \hat{\mathbf{R}}_{\mathbf{y} \psi}(\mathbf{c}(\theta) \operatorname{od}(\theta))^{*}}{\|\mathbf{e}(\theta) \operatorname{of}(\theta)\|^{2}(\mathbf{c}(\theta) \operatorname{od}(\theta))^{T} \hat{\mathbf{R}}_{\psi \psi}(\mathbf{c}(\theta) \operatorname{od}(\theta))^{*}}
\end{aligned}
$$

with

$$
\hat{\mathbf{R}}_{\mathbf{y} \psi}=(1 / Q) \sum_{q=1}^{Q} \mathbf{y}(q) \boldsymbol{\psi}^{H}(q)
$$




$$
\hat{\mathbf{R}}_{\psi \psi \psi}=(1 / Q) \sum_{q=1}^{Q} \boldsymbol{\psi}(q) \boldsymbol{\psi}^{H}(q)=\mathbf{I}_{M \times M}
$$

where $\|$.$\| is the of operator Euclidean norm, \hat{\beta}(\theta)$ is the least squares (LS) estimation of the radar reflection coefficient or the magnitude of the complex amplitude of the target in the $\theta$ direction, for $q=1,2, \ldots, Q$ is the index of the data sample. The number of detected targets can be identified from the peaks of the spatial spectrum in the LS estimation.

\section{RESULTS AND DISCUSSION}

\section{A. Virtual Array}

The calculation of the VA size $(E)$ in the SMIMO radar can apply the concept of VA in the MIMO radar. It was conducted by expanding the elements in a single antenna to radiate orthogonal signals; thereby, they could transform into nonoverlapping subarray elements or NOES. The VA is the convolution result of Tx and Rx arrays [7], precisely between the Tx beampattern and Rx beampattern. In determining the $\mathrm{VA}$, the subarray was considered a single antenna in the center of the location to radiate orthogonal signals following the rules as proposed in [7].

Based on consideration of the comparison between PMIMO-Tx and the four OES, the following is the example of the VA calculation for the SMIMO $\operatorname{radar}(M=N=8)$ with four NOES, in which the number of the elements is two for each subarray.

$$
\begin{aligned}
& \text { Array } T x=\left\{\begin{array}{llllllll}
0 & 1 & 0 & 1 & 0 & 1 & 0 & 1
\end{array}\right\} \text {; } \\
& \text { Array } R x=\left\{\begin{array}{llllllll}
1 & 1 & 1 & 1 & 1 & 1 & 1 & 1
\end{array}\right] \text {; } \\
& \mathrm{VA}=\left\{\begin{array}{lll}
1 & 12233443322110
\end{array}\right\}
\end{aligned}
$$

Since there are four NOES, one element location, which value is 1 , is shown in different font colors. The Rx array was 1 at eight element locations. The VA for SMIMO radar with NOES was 14; it was almost close to the size of the VA for MIMO radar with 15.

The following is the comparison for SMIMO radar with two NOES in which each subarray has four elements.

$$
\begin{aligned}
& \text { Array } \mathrm{Tx}=\left\{\begin{array}{llllllll}
0 & 0 & 1 & 0 & 0 & 0 & 1 & 0
\end{array}\right\} \text {; } \\
& \text { Array } \mathrm{Rx}=\left\{\begin{array}{llllllll}
1 & 1 & 1 & 1 & 1 & 1 & 1 & 1
\end{array}\right\} \text {; }
\end{aligned}
$$

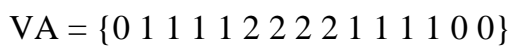

therefore, the VA size for SMIMO radar with 2 NOES was 13.

Reference [7] suggests that each waveform in radar PMIMO-TX, as proposed in [8] and utilized radiation elements in the form of Tx OES, is radiated from the center of phase or element location of the antenna in the subarray with $M_{K}=(M-K+1)$. As a result, the central element was 1 and the others were 0 . The following examples show the calculation of the VA for every PMIMO-Tx radar with $M=N$ $=8$. For instance, the configuration of $K=4$, thereby $M_{K}=8-$ $4+1=5$. The followings are Tx, Rx, and VA arrays.

$$
\begin{aligned}
& \text { Array } \mathrm{Tx}=\left\{\begin{array}{llllllll}
0 & 0 & 1 & 1 & 1 & 1 & 0 & 0
\end{array}\right\} \text {; } \\
& \text { Array } R x=\left\{\begin{array}{llllllll}
1 & 1 & 1 & 1 & 1 & 1 & 1 & 1
\end{array}\right\} \text {; } \\
& \mathrm{VA}=\{0111112222211111100\}
\end{aligned}
$$

Since there were four subarrays in which the number of elements was five for each, the element located in the middle of each subarray was one (it was considered as an antenna); it is shown in different font colors. Meanwhile, the value of the Rx array was 1 in eight element locations. The size of the VA for the PMIMO radar was 11 and located in between the two extreme configurations, which was slightly higher than the PA radar with 8 and lower than the MIMO radar with 15 .

In comparing the size of the VA in the SMIMO radar with the OES and NOES, Table I displays the number of OES and NOES subarrays, the elements for each subarray, the number of OES/NOES, and the number of VA size. The number of overlapping locations in the SMIMO OES radar influences the VA due to the simultaneous use of several elements among subarrays; thus, they generated signals that were not perfectly orthogonal. It is shown in the configuration 2 OES which had 6 element locations which $O$ with $E$ was 9, almost close to $E$ in the PA radar. On the other hand, if the location of the element $O$ was small as in the configuration $7 \mathrm{OES}$ with one $O$ element, then the $E$ radar was one less than MIMO radar. Although, in general, the PMIMO-Tx radar based on OES has a high SINR [8] because the number of elements in the OES is large, it increases the directional gain such as a PA radar. Therefore, to adjust to the desired target radar conditions, it is necessary to compromise between $E$ which is proportional to angular resolution, angular accuracy, and the number of target detections toward the number of the OES.

The calculation of $E$ in the FPMIMO/PMIMO-Tx-Rx was conducted in [13], [14] where the Tx and Rx were the OES. Therefore, according to [7], each signal was radiated from the middle point of the phase or the location of the antennas' element at the subarray in the Tx with $M_{K}=(M-K+1)$. Hence, the middle element was 1 , while the others would be 0 ; the same condition applied to the subarray in the $\mathrm{Rx}$, which element number was $N_{L}=(N-L+1)$. As an example, the calculation of $E$ was $K=L=4$ and $M=N=8$, therefore $M_{K}=$ $N_{L}=5$. Tx, Rx, and VA arrays can be expressed as follows.

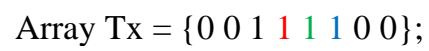

$$
\begin{aligned}
& \text { Array } R x=\left\{\begin{array}{llllllll}
0 & 0 & 1 & 1 & 1 & 1 & 0 & 0
\end{array}\right\} \text {; }
\end{aligned}
$$

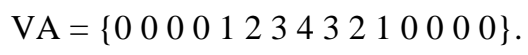

Since there are four subarrays in the $\mathrm{Tx}$ and $\mathrm{Rx}$, the arrangement of the elements is similar. The $E$ for FPMIMO radar was 7 since there were only seven element locations out of 15 locations that were higher than 1 .

Table II presents the result of $E$ from all configuration combinations built for $M=N=8$ using (9) with $1 \leq K \leq M$ and $1 \leq L \leq N$. The table shows that the value of $E$ from subarray configurations in the Tx and Rx for SMIMO radar, such as PMIMO-Tx and FPMIMO/PMIMO-Tx-Rx, is between the value of $E$ for interrelated PA and MIMO radars, 
TABLE I

COMPARISON OF THE NUMBER ELEMENT OF ViRTUAL ARRAYS $(E)$ FOR THE VARIATION OF THE SMIMO RADAR WITH $M=N=8$

\begin{tabular}{|c|c|c|c|c|c|}
\hline \multicolumn{2}{|c|}{ Number of SAs } & \multirow{2}{*}{$\begin{array}{c}\text { Element Number of per-SA } \\
4\end{array}$} & \multirow{2}{*}{$\begin{array}{c}\text { Number of O Location in SA } \\
0\end{array}$} & \multirow{2}{*}{$\frac{\text { Number of NO Location in SA }}{2}$} & \multirow{2}{*}{$\begin{array}{c}\boldsymbol{E} \\
13 \\
\end{array}$} \\
\hline \multirow{3}{*}{ NOES } & 2 & & & & \\
\hline & 4 & 2 & 0 & 4 & 14 \\
\hline & 8 & 1 & 0 & 8 & 15 \\
\hline \multirow{6}{*}{ OES } & 2 & 7 & 6 & 2 & 9 \\
\hline & 3 & 6 & 5 & 2 & 10 \\
\hline & 4 & 5 & 4 & 2 & 11 \\
\hline & 5 & 4 & 3 & 2 & 12 \\
\hline & 6 & 3 & 2 & 2 & 13 \\
\hline & 7 & 2 & 1 & 2 & 14 \\
\hline \multicolumn{6}{|l|}{ Note: } \\
\hline $\begin{array}{ll}\mathrm{SA} & : \\
\mathrm{O} & : \\
\mathrm{NO} & :\end{array}$ & $\begin{array}{l}\text { rray } \\
\text { lap }\end{array}$ & $\begin{array}{l}\text { OES } \\
\text { NOES }\end{array}$ & $\begin{array}{l}\text { overlapped equal subarray } \\
\text { non-overlapped equal subarray }\end{array}$ & & \\
\hline
\end{tabular}

TABLE II

VARIATION OF $E$ FOR TX-RX ARRAYS IN THE SMIMO RADAR WITH $M=N=8$

\begin{tabular}{|c|c|c|c|c|c|c|c|c|c|}
\hline \multirow{2}{*}{\multicolumn{2}{|c|}{$\boldsymbol{E}$}} & \multicolumn{8}{|c|}{$\boldsymbol{L}$} \\
\hline & & 1 & 2 & 3 & 4 & 5 & 6 & 7 & 8 \\
\hline \multirow{8}{*}{$K$} & 1 & 1 & 2 & 3 & 4 & 5 & 6 & 7 & 8 \\
\hline & 2 & 2 & 3 & 4 & 5 & 6 & 7 & 8 & 9 \\
\hline & 3 & 3 & 4 & 5 & 6 & 7 & 8 & 9 & 10 \\
\hline & 4 & 4 & 5 & 6 & 7 & 8 & 9 & 10 & 11 \\
\hline & 5 & 5 & 6 & 7 & 8 & 9 & 10 & 11 & 12 \\
\hline & 6 & 6 & 7 & 8 & 9 & 10 & 11 & 12 & 13 \\
\hline & 7 & 7 & 8 & 9 & 10 & 11 & 12 & 13 & 14 \\
\hline & 8 & 8 & 9 & 10 & 11 & 12 & 13 & 14 & 15 \\
\hline
\end{tabular}

namely $E_{\mathrm{PA}}<E_{\mathrm{SMIMO}}<E_{\mathrm{MIMO}}$. The description also shows that the SMIMO radar with the number of subarrays in the Tx and $\mathrm{Rx}$, namely $K$ and $L$, is a general form of orthogonal signal radar. The special conditions of the SMIMO radar are the PA or SMIMO OES radar $(K=1, L=8)$, MIMO or SMIMO NOES $(K=L=8)$, and PMIMO-Tx or SMIMO OES $(1<K<M, L=8)$.

If $d_{M}$ and $d_{N}$ were not $d$ or greater than $d$, the result of $E$ would be greater than what is listed in Table II. Moreover, the $d_{M}=L d_{N}$ and $d_{N}=d$ would yield $E$ maximum, namely $K L$. For example, for $M=N=8$, the $E$ maximum for the PA, MIMO, PMIMO-Tx, dan FPMIMO/PMIMO-Tx-Rx radars was 8, 64, 32, dan 16, respectively. As for the number of $M$ and $N$ elements, including the space between the elements, the obtained $E$ in the MIMO, PMIMO-Tx, and PMIMO-Tx-Rx radars was 11,6 , and 4 times greater than $E$ resulted from the PA radar [14]. In fact, $M=N$, which has been specified, to adjust the distance between elements in the Tx and Rx to get a bigger $E$ can only be implemented manually since the location of the antenna of Tx and Rx arrays have been specified from the beginning.

\section{B. Angular Resolution}

The forming of the VA is caused by the Tx array transmitting orthogonal signals to form a more focused beam pattern. It happens because of the formation of a larger array in the VA. Beampattern output, which is the overall beampattern, is the multiplication between the $\mathrm{Tx}$ and $\mathrm{Rx}$ arrays. Furthermore, the beampattern in the Tx is a power pattern radiated by $\mathrm{Tx}$ antennas in all target directions. Likewise, the Rx beampattern is a power pattern received from a target as a function of the angle of arrival of the echo signals. Nonetheless, it will be difficult to determine the angle of approach if $\mathrm{Rx}$ only utilizes a single antenna since the stronger signal from the top of the beam and the weaker signal from the sidelobe will be hard to be distinguished. However, it will not happen if $\mathrm{Rx}$ is an array so that the beam can be digitally directed to the angle of arrival of the echo signals.

The beampattern of $\mathrm{Tx}, \mathrm{Rx}$, and its overall would be numerically simulated to show the connection between VA and angular resolution in the SMIMO radar. The SMIMO radar with $M$ and $N$ elements has the advantage of adjusting the configuration of the Tx-Rx ( $K$ and $L$ ) subarrays, i.e. OES, OUS, NOES, NOUS, etc., based on conditions of the target to be detected. Fig. 2 shows the performance of the beampattern of those radars with $M=N=8, K=4$, and $L=5$ as the cases. The configurations of Tx and Rx subarrays, namely $K=4$ and $L=5$, represent a particular condition of the subarray, that is OES for various conditions of the distance between elements in the Tx and Rx arrays, namely $d_{M}$ and $d_{N}$.

In Fig. 2(a), the beampattern in the SMIMO OES radar for $K=4, L=5$, and $M=N=8$ also the distance between elements in the Tx and $\mathrm{Rx}$ arrays is $0.5 \lambda$ where $\lambda$ is the wavelength from the carrier signals. The overall beampatern resembles the beampattern in the Rx. However, if the distance 


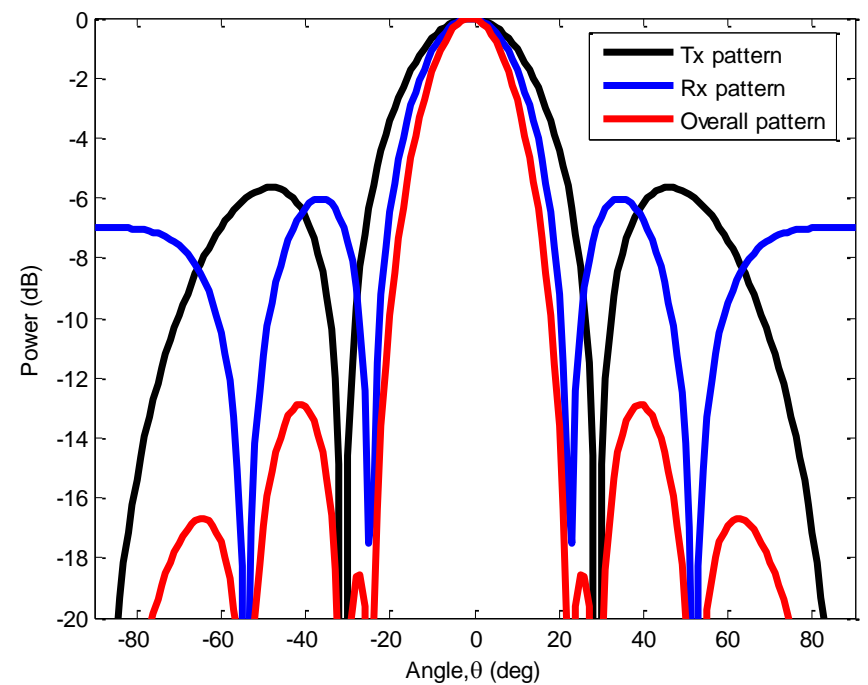

(a)

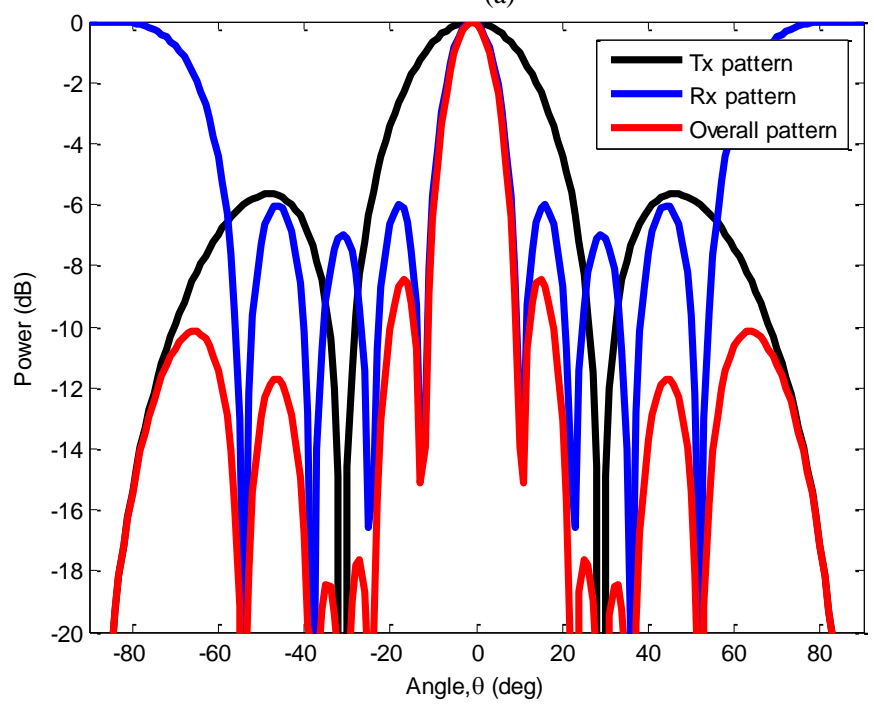

(c)

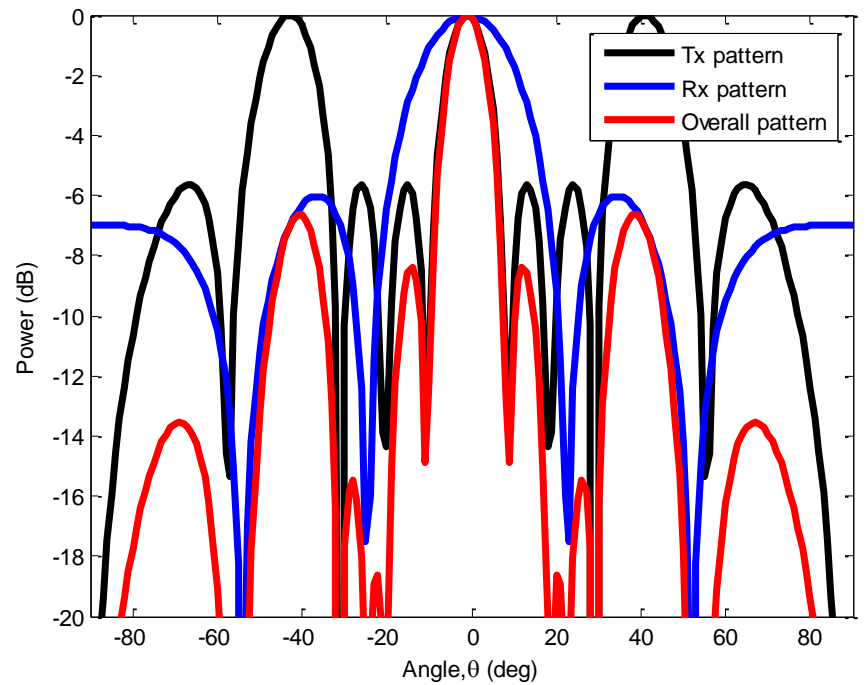

(b)

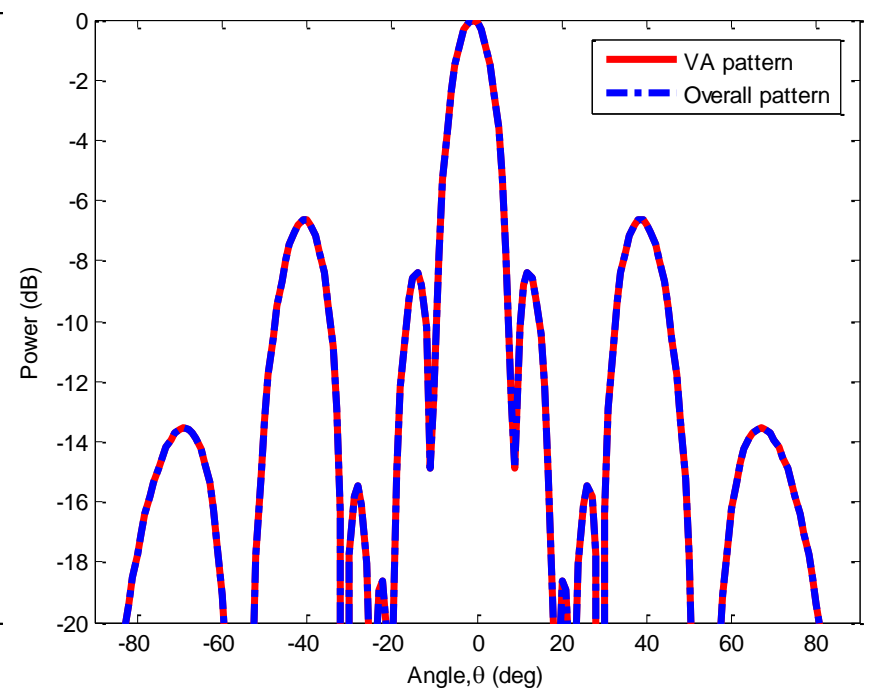

(d)

Fig. 2 Beampattern in the SMIMO radar with $K=4, L=5, M=N=8$, and $d=0.5 \lambda$ for: (a) $d M=d N=d,(b) d M=3 d$, $d N=d$, (c) $d M=d$, $d N=2$, and (d) the comparison between the VA and overall patterns.

between the radar elements in the Tx array was enlarged to three times greater than the $d_{N}$, the obtained beampattern is shown in Fig. 2(b). The obtained overall beampatterns had narrow beams. Moreover, the grating lobes that occurred in the Tx beampattern did not appear in the overall beampattern. The results of beampattern in Fig. 2 are almost similar to Fig. 2(b). The differences lay in the distance between elements in the Tx and Rx. The distance between elements in the Tx was $0.5 \lambda$, while in the $\mathrm{Rx}$, it was two times greater than $d_{M}$. Hence, the beampattern output can be denoted as the multiplication between beampatterns in the Tx and Rx; in other words, the beampattern output is the VA beampattern as in Fig. 2(d). It indicates that by determining the correct geometry/distance between elements in Tx and Rx arrays, the angular resolution of this radar system will increase without adding any antenna elements to the array.

A high gain beam was obtained in the PA transmission signal; it increased the SNR by $M$ factor times. However, it did not occur in the orthogonal signal transmission. Therefore, it is necessary to compromise between signal transmission with directional gain and orthogonal signal transmission to obtain an angular resolution that suits the target conditions. It supports the application of the SMIMO radar concept because it guarantees a compromise of the two extreme configurations so that it can simultaneously provide SNR, angular resolution, the maximum number of target detection, and beampattern.

There is potential for using subarrays with different elements, namely unequal subarray (US) and to overcome the limitations of equal subarray, namely equal subarray (ES). Significantly if it is adjusted to the conditions of multi-targets because, with high directional gain, it is suitable for targets having greater range and weak/small radar cross-section (RCS). If the target condition varies i.e., in range and RCS, the use of a combination of varied subarray configurations (OS/NOS and ES/US) can be realized. As an example, nonoverlapped equal subarray (NOES), overlapped equal subarray (OES), non-overlapped unequal subarray (NOUS), or overlapped unequal subarray (OUS) can be realized at the 


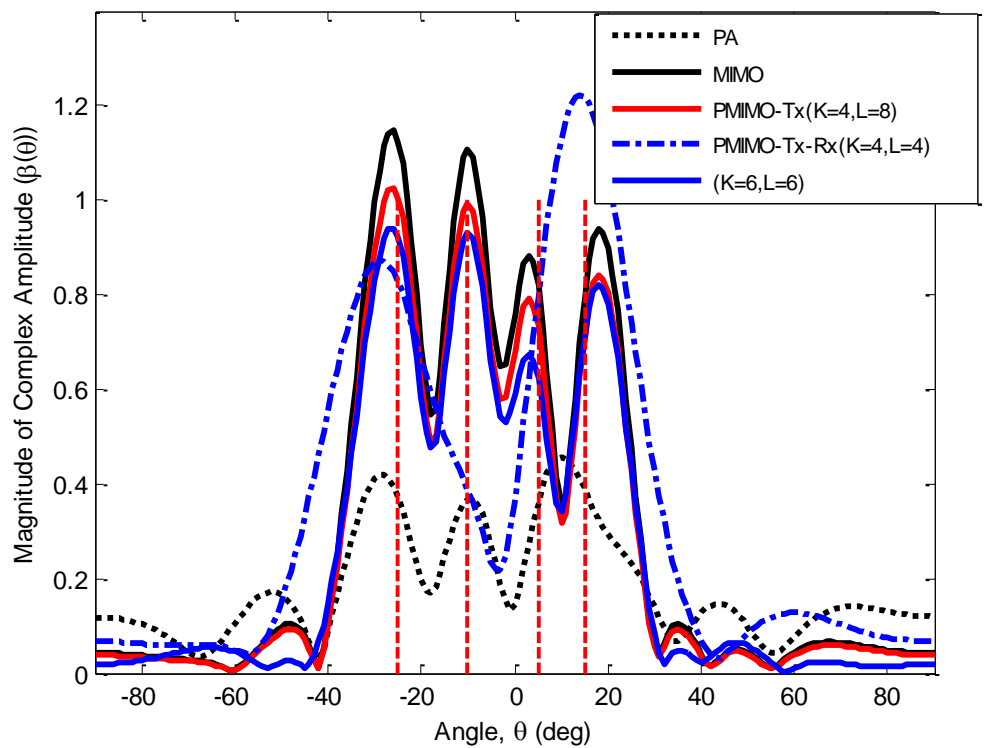

Fig. 3 Magnitude of the complex amplitude as a comparison of the accuracy and resolution of the target angle with $\theta=\left\{-25^{\circ},-10^{\circ}, 5^{\circ}, 15^{\circ}\right\}, \beta(\theta)=1$, and $M=$ $N=8$ for various radar configurations.

same time and on one Tx array. However, there is also a need for technical considerations in its implementation, which will determine whether or not it can be applied practically, especially to the waveform generator in the Tx and signal processor in the Rx for the application of the subarray method.

\section{The Connection between Virtual Arrays and Maximum Detected Number of Targets}

Chapter IV-A presented the connection between the numerical calculation of the VA using (9) as in the previous study [16]. The VA was proportional toward the angular resolution and accuracy. Thus, it would also be proportional toward the determination of the maximum number of target detection $\left(T_{\max }\right)$. The calculation of the maximum number of targets in the MIMO radar has been presented in [16, eq. (22)]. This calculation was valid and could be employed to calculate the maximum target detections to the PA, PMIMOTx, FPMIMO/PMIMO-Tx-Rx radars, which were special conditions for the SMIMO radar using (10). As evidence, numerical results were obtained by using (11) as in Fig. 3 . Therefore, Fig. 3 shows the comparison of the four parameters for all variations of the SMIMO OES radar with $M=N=8$.

In Fig. 3, the MIMO radar $(K=L=8)$, PMIMO-Tx radar ( $K=4, L=8$ ), and the configuration of subarrays ( $K=6, L=$ 6) are able to detect the four targets at $\theta=\left\{-25^{\circ},-10^{\circ}, 5^{\circ}\right.$, $\left.15^{\circ}\right\}$. On the other hand, PA radar $(K=1, L=8)$ and PMIMOTx-Rx $(K=4, L=4)$ can only detect two targets at $\theta=\left\{-25^{\circ}\right.$, $\left.-10^{\circ}\right\}$. Among the four radars, the MIMO radar has the best detection accuracy. The MIMO radar also provides the best detection accuracy when detecting the magnitude of the complex amplitude of the echo signal. It is related to the VA size of the MIMO radar, which is the largest, thus increasing its angular detection accuracy and angular resolution.

Based on the $T_{\max }$ expression for the SMIMO radar in (10), the $T_{\max }$ value of the PMIMO-Tx and PMIMO-Tx-Rx radar signal configurations was between the $T_{\max }$ values for the PA and MIMO radars which were related $T_{\max }$, namely $T_{\max , \mathrm{PA}}<$
$T_{\text {max,PMimo }}<T_{\max , \text { MIMO. }}$ As an example, for Tx-Rx with $M=N$ $=8$, the results for $T_{\max , \text { PA }}, T_{\max , \text { MIMO }}, T_{\max , \text { PMIMO-Tx }}$, and $T_{\text {max,PMiMO-Tx-Rx }}$ were $4,(7,32),(5,16)$, and $(3,8)$, respectively. Therefore, referring to Fig. 3, the MIMO and PMIMO radars can adequately detect the four targets since it is still within the limits of the radar detection capability, which is greater than 4 .

\section{CONCLUSION}

This paper discussed the size of the VA, angular resolution, maximum number of detected targets, and magnitude of the complex amplitude of the echo signal in the SMIMO radar with various subarray conditions. The size of the VA determines the angular resolution. On the other hand, the number of detected targets is influenced by the subarray condition, such as OES, OUS, NOES, dan NOUS. In the SMIMO radar with $M$ and $N$ as the total number of antennas in the Tx array, the performance parameter of the SMIMO radar is determined by the size of the subarrays in Tx-Rx, namely $K$ and $L$. The expressions of the VA size, the maximum number of detected targets, and the magnitude of the complex amplitude depend on the number of subarrays in Tx-Rx of SMIMO radars, PA, MIMO PMIMO-Tx, and PMIMO-Tx-Rx radars are the particular conditions. The adaptive, flexible, and programmable changes to the $K$ and $L$ values in the SMIMO radars provides multi-target detection capabilities in an increasingly reliable and flexible multiantenna radar system that can adapt to the conditions of the target and its environment. It also facilitates the planning of the radar design, primarily when implemented using a programmable platform for radar system design such as Software Defined Radio (SDR) or USRP, etc.

\section{REFERENCES}

[1] I. Bilik, O. Longman, S. Villeval, and J. Tabrikian, "The Rise of Radar for Autonomous Vehicles: Signal Processing Solutions and Future 
Research Directions," IEEE Signal Processing Magazine, Vol. 36, No. 5, pp. 20-31, Sep. 2019.

[2] R.C. Hansen, Phased Array Antennas, 2nd ed. New Jersey, USA: John Wiley \& Sons, 2009.

[3] D.S. Zrnic, G. Zhang, and R.J. Doviak, "Bias Correction and Doppler Measurement for Polarimetric Phased-Array Radar," IEEE Transaction on Geoscience and Remote Sensing, Vol. 49, No. 2, pp. 843-853, Feb. 2011.

[4] A. Haimovich, R. Blum, and L. Cimini, "MIMO Radar with Widely Separated Antennas," IEEE Signal Processing Magazine, Vol. 25, No. 1, pp. 116-129, Jan. 2008.

[5] Y. Li, H. Ma, Y. Wu, L. Cheng, and D. Yu, "DOA Estimation for Echo Signals and Experimental Results in the AM Radio-Based Passive Radar," IEEE Access, Vol. 6, pp. 73316-73327, Nov. 2018

[6] X. Song, N. Zheng, and T. Bai, "Resource Allocation Schemes for Multiple Targets Tracking in Distributed MIMO Radar Systems,' International Journal of Antennas and Propagation, Vol. 2017, pp. 1-12, Jul. 2017

[7] M.S. Davis and A.D. Lanterman, "Coherent MIMO Radar: the Phased Array and Orthogonal Waveforms," IEEE Aerospace and Electronic Systems Magazine, Vol. 29, No. 8, pp. 76-91, Aug. 2014.

[8] A. Hassanien and S.A. Vorobyov, "Phased-MIMO Radar: A Tradeoff between Phased-Array and MIMO Radars," IEEE Transactions on Signal Processing, Vol. 58, No. 6, pp. 3137-3151, Jun. 2010.

[9] W. Khan, I.M. Qureshi, A. Basit, and M. Zubair, "Hybrid Phased MIMO Radar with Unequal Subarrays," IEEE Antennas and Wireless Propagation Letters, Vol. 14, pp. 1702-1705, Apr. 2015.
[10] S. Tahcfulloh and G. Hendrantoro, "Phased-MIMO Radar using Hadamard Coded Signal," Proceeding of IEEE International Conference on Radar Antenna Microwave Electronics and Telecommunications, Tangerang, Indonesia, Oct. 2016, pp. 13-16.

[11] A. Alieldin, Y. Huang, and W.M. Saad, "Optimum Partitioning of a Phased-MIMO Radar Array Antenna," IEEE Antennas and Wireless Propagation Letters, Vol. 16, pp. 2287-2290, Aug. 2017.

[12] M. Hardiwansyah, S. Tahcfulloh, and G. Hendrantoro, "Parameter Identifiability of Phased-MIMO Radar," Proceeding of International Conference on Artificial Intelligence and Information Technology, Yogyakarta, Indonesia, Mar. 2019, pp. 192-195.

[13] S. Tahcfulloh and G. Hendrantoro, "Full Phased MIMO Radar with Colocated Antennas," International Journal on Communications Antenna and Propagation, Vol. 9, No. 2, pp. 144-154, Apr. 2019.

[14] S. Tahcfulloh and G. Hendrantoro, "FPMIMO: General MIMO Structure with Overlapping Subarrays for Various Radar Applications," IEEE Access, Vol. 8, No. 1, pp. 11248-11267, Jan. 2020.

[15] A.F. Utami, Iswandi, and I.W. Mustika, "Random Delay Mitigation in Pulse Radar Implementation on Universal Software Radio Peripheral (USRP)," International Journal on Information Technology and Electrical Engineering, Vol. 2, No. 3, pp. 85-90, Sep. 2018.

[16] J. Li, P. Stoica, X. Luzhou, and W. Roberts, "On Parameter Identifiability of MIMO Radar”, IEEE Signal Processing Letters, Vol. 14, No. 12, pp. 968 - 971, Dec. 2007. 